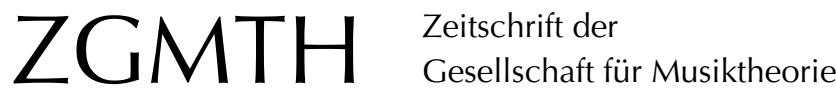

Fasshauer, Tobias (2009): Markus Roth, Der Gesang als Asyl. Analytische Studien zu Hanns Eislers Hollywood-Liederbuch, Hofheim: Wolke 2007. ZGMTH 6/1, 177-181. https://doi.org/10.31751/401

(C) 2009 Tobias Fasshauer

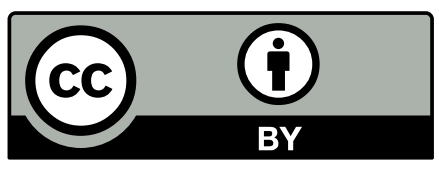

Dieser Text erscheint im Open Access und ist lizenziert unter einer Creative Commons Namensnennung 4.0 International Lizenz.

This is an open access article licensed under a Creative Commons Attribution 4.0 International License.

veröffentlicht / first published: 15/02/2009

zuletzt geändert / last updated: 27/02/2009 


\section{Markus Roth, Der Gesang als Asyl. Analytische Studien zu Hanns Eislers Hollywood-Liederbuch, Hofheim: Wolke 2007}

»Man muss sich endlich dazu entschließen, Musik als eine von den Menschen für die Menschen in bestimmten konkreten gesellschaftlichen Verhältnissen entstehende und vergehende Kunst anzusehen." Hanns Eislers Diktum von 1932 wirkt heute, angesichts des besinnungslosen Strukturfetischismus, der in so vielen musiktheoretischen Analysen obwaltet, bemerkenswert aktuell. Bei der Analyse von Eislers eigener Musik erscheint es allemal angebracht, die verwendeten musikalischen Ausdrucksmittel auf ihre historische und soziale Bedeutungsprägung zu befragen, ohne andererseits den inneren $\mathrm{Zu}$ sammenhang der Werke zu vernachlässigen und womöglich dem Schematismus einer vulgarisierten Figurenlehre zu verfallen. Einen solchen Vorstoß unternimmt in begrüßenswerter Weise Markus Roth mit seiner 2007 vorgelegten Studie über Eislers HollywoodLiederbuch, jene als Zyklus nie verbindlich fixierte Sammlung von Klavierliedern, die, in Roths Worten, mit ihrer »eigenartigen Synthese unterschiedlichster stilistischer und kompositionstechnischer Momente als Brennpunkt des Eislerschen Exilschaffens angesehen werden kann." (15) Mit seinem betont analytisch orientierten Ansatz versucht Roth, die Auseinandersetzung mit Eisler aus dem Sumpf politischer Grabenkämpfe herauszuholen und zu seiner kritischen Würdigung als Komponist vorzudringen. Dabei vertritt er die Überzeugung, dass die kompositionstechnische Seite bei Eisler nur sauf der Grundlage der in den Schriften und Lehrwerken" Arnold Schönbergs "entfalteten Begriffe und Denkweisen« zu erschließen ist - Schönberg war bekanntlich Eislers Lehrer. Roths Analysen bewegen sich zwischen drei Schwerpunkten: erstens der Untersuchung motivischer Beziehungen nach Maßgabe der Schönbergschen Kategorie der `Grundgestalt` (im Sinne einer moti- vischen Ausgangskonfiguration, auf die sich die weiteren Gestalten einer Komposition zurückführen lassen), zweitens der Betrachtung von Tonalität und Harmonik und drittens der Vermittlung kompositorischer mit inhaltlichen Momenten.

Es bezeichnet die Redlichkeit von Roths Argumentation, dass er sich über die jeweiligen Probleme seiner Methoden stets Rechenschaft ablegt, so auch über jene der GrundgestaltAnalyse. "Strukturelle Mehrdeutigkeiten auf unterster syntaktischer Ebene", weiß der Autor, »liegen in der Natur der Sache« (28); der Versuch ihrer umfassenden Einordnung mündet leicht in Beliebigkeit. So warnt Roth ausdrücklich davor, bei der Grundgestalt-Analyse »das Wesentliche vor lauter Details aus den Augen « und sich in einer »kleingliedrigen und letztlich abstrakten Denkweise« zu verlieren (28f., 31). Belege für diese Gefahren lassen sich - als Randerscheinungen, wohlgemerkt - auch in seinen eigenen Analysen ausmachen. Verwiesen sei etwa auf einen an sich harmlosen, aber erhellenden Lapsus bei der Untersuchung des frühen Eisler-Liedes Ach, es ist so dunkel, die Roth zur einleitenden Exemplifizierung der Grundgestalt-Analyse dient. Dort wird für die Tonfolge cis-dis-c in Takt $2 \mathrm{f}$. die Erklärung als transponierte Umkehrung der Töne 3-5 der Grundgestalt (gis-fis-a, Takt $1 \mathrm{f}$.) angeboten (27f.). In der als Notenbeispiel reproduzierten Druckausgabe steht allerdings nicht cis-dis-c, sondern cis- $d-c$. Falls es sich hier um einen Fehler der Ausgabe handelt, hätte dies zumindest Erwähnung finden müssen; im Übrigen steht der Wert der Analysemethode selbst in Frage, wenn strukturell vermeintlich Konstitutives und Arbiträres so dicht beieinander liegen. Die vom Autor freimütig eingeräumten Schwierigkeiten der Grundgestalt-Analyse spiegeln sich auch in der Vagheit und Unverbindlichkeit man- 
cher Formulierung: Da »dürften« bestimmte Außenstimmenkonturen im Beziehungsgefüge »von Bedeutung" sein (182f.), ist ein festgehaltener Ton im Diskant "vermutlich eine Entsprechung « zu einem Stehenbleiben der Bassstimme an anderer Stelle (162) oder stammen charakteristische Merkmale einer Gestalt »augenscheinlich" aus einer anderen (233). Indessen spielt Grundgestalt-Analyse in den Untersuchungen eine weit geringere Rolle als zu Beginn der Studie suggeriert wird; als wesentlicher erweist sich ein kritisch reflektierter, dem jeweiligen Gegenstand angepasster »Methodenpluralismus« (32), der überwiegend zu sehr überzeugenden Ergebnissen führt. Roths flexible Herangehensweise eröffnet dabei auch Einsichten in Eisler-typische, vom jeweiligen Beziehungsgefüge im Prinzip unabhängige Verfahren, wie etwa die vom Autor so bezeichnete 'Geländertechnikı, die Strukturbildung durch Verflechtung schromatischer Fäden<.

Zu den größten Stärken von Roths Arbeit gehören seine Einlassungen zu Tonalität und Harmonik, wenn auch seine Ablehnung diesbezüglicher bisheriger Interpretationsansätze der Eisler-Forschung ein wenig zu apodiktisch gerät. So übt er scharfe Kritik an der zuerst von Jürgen Elsner formulierten Auffassung, dass bei Eisler eine sPhrygisierung، funktionaler Harmonik stattfinde; dieser Betrachtungsweise, meint Roth, liege ein "verschwommener Begriff von Modalität zugrunde, mit dem zwangsläufig eine Überbewertung angeblich modaler Aspekte einhergeht« (109). Doch so sehr der Vorwurf begrifflicher Unschärfe hier zutreffen mag, so wenig lässt sich andererseits von der Hand weisen, dass eine Verselbständigung des sogenannten phrygischen Halbschlusses, der als solcher bereits keine modale Erscheinung mehr darstellt, zumindest in Eislers Kampflied- und Song-Harmonik zu den auffälligsten Charakteristika zählt. Der Aufweis einer einzigen von Elsner fehlinterpretierten Stelle (Anm. 324) ändert daran nichts.

Auch in Bezug auf Tonalität und Harmonik vertritt Roth die These, dass Eislers Denken von Schönberg her zu begreifen sei, und zwar betrifft dies das Konzept der salten Tonalität als eines zentrierten Systems ebenso wie Schönbergs Diskussion der schwebenden Tonalität sowie der Quarten- und Ganztonharmonik in der 3. Auflage seiner Harmonielehre. Die Beziehungen der Harmonik Eislers zur Theorie Schönbergs werden in vielen Analysen plausibel nachvollziehbar gemacht; andererseits versäumt der Autor nicht, auf die große Bedeutung smechanischer Verfahrensweisen für Eisler einzugehen, in denen eine neusachliche Gegentendenz zur Prägung durch Schönberg greifbar wird.

Besondere Aufmerksamkeit verdient Roths Auseinandersetzung mit Eislers Harmonik dadurch, dass er im Wesentlichen auf dieser Ebene auch einen Aspekt behandelt, dem bei einem Komponisten mit so ausgeprägtem historischen und politisch-gesellschaftlichen Bewusstsein eine zentrale Bedeutung zukommt: das Verhältnis und Verhalten zu überkommenen musikalischen Idiomen, darunter insbesondere den populären. (Es ist gerade dieser Komplex, der sich dem Zugang über eine rein positivistisch strukturorientierte Analysemethode schlechterdings verschließt.) Bei der Interpretation der spopulären` Anklänge im Hollywood-Liederbuch argumentiert der Autor einmal mehr überaus vorsichtig und differenziert: Zum einen befindet er es für schwierig, Eislers »Anverwandlung des Populären im Sinne einer `Musik über Unterhaltungsmusik» an konkreten Musterbeispielen aus dem Jazz-, Schlager-, Revue- oder gar Operettenbereich im Detail zu belegen«; »[e]ingehende analytische Studien zu diesem Problemkreis« stünden bislang noch aus (192). (Wie dringend in der Tat etwa eine kritische Geschichte der Jazz-Harmonik einmal geschrieben müsste, zeigt sich auf Seite 113, wo der Autor lapidar äußert, eine »Häufung ajoutierter Septimen« im Vielleicht-Lied aus Eislers Bühnenmusik zu Die Rundköpfe und die Spitzköpfe suggeriere »eine zumindest latente Nähe zum Jazz« - in einem Stück von 1934, aus einer Zeit also, in der im Jazz, und zumal in dem von Eisler rezipierten, solche sfreien`, d. h. weder in Quintfortschreitungen noch in Mixtursätze eingebundenen Septakkorde vermutlich nicht leicht zu finden sein dürften, von der blue sev- 
enth und eventuell noch der tonikalen major seventh einmal abgesehen.)

Auf der anderen Seite vermeidet Roth, die Anwendung der sabgesunkenes 19. Jahrhundert s signalisierenden harmonischen Mittel einseitig und schlicht nur im parodistischen Sinn zu deuten: "Natürlich ist die Sphäre des Leichten, `Populären` bei Eisler nicht durchweg negativ besetzt; insgesamt kann seine Haltung zur Unterhaltungsmusik als eine durchaus zwiespältige gelten.« (197) Entscheidend sei die Funktion der Mittel im Kontext sich wechselseitig kommentierender heterogener Stilebenen: "Ziel ist nicht die Freilegung verschütteter Ausdrucksgehalte" - Roth verweist hier auf Ernst Kreneks >Ursinn «-Theorie -, „sondern ihre Kontextualisierung, ihre unerwartete Platzierung, ihr Einsatz zur Hervorrufung von Brechungen." (108) Mit solchen, von Roth anschaulich aufgeschlüsselten `Verfremdungen im Großen korrespondieren satztechnische Manipulationen an konventionell verfestigten Materialkonfigurationen auf der Detailebene, die der Autor aber wohlweislich nicht als Verfremdungs-, sondern als ,Verzerrungstechniken bezeichnet (weder von Eisler noch von Brecht wurde der Verfremdungsbegriff jemals im kompositionstechnischen Sinne verwendet). Insofern derartige Verfahren auch für die >Brecht-Komponisten W Weill und Dessau sehr typisch sind, hätte man sich ein ausführlicheres Eingehen darauf gewünscht; leider fällt Roths Beschäftigung mit innen aber so knapp aus, dass die Vorstellung nahegelegt wird, es handle sich in der Regel um nur äuBerliche, punktuelle Eingriffe (hauptsächlich in Gestalt mehr oder minder willkürlicher Alterationen und Ajoutierungen von Tönen) in vorgegebene Satzmuster, und nicht um Maßnahmen, die mit Eislers sonstigen Verfahren integral in Verbindung stehen.

Wer sich mit dem Phänomen stilistischer Heterogenität und der Verwendung salter Tonalität bei Eisler auseinandersetzt, kommt an der in der einschlägigen Literatur viel zitierten These, dass in der Neuen Musik »Material und Verfahrensweise auseinandertreten, und zwar in dem Sinn, dass das Material der Verfahrensweise gegenüber relativ gleichgültig wird", schwerlich vorbei. Diese Formulierung, enthalten in dem von Eisler und Theodor W. Adorno in den vierziger Jahren verfassten Buch Komposition für den Film, eröffnet, in den Worten Roths, »eine Hintertür, die Verwendung scheinbar zerschlissener resp. süberholter`Materialien durch den Verweis auf die Avanciertheit neuer Verfahrensweisen zu adeln« (167). Da zumindest der Wortlaut der Material-Verfahrensweise-These offenbar auf Adorno zurückgeht, widersteht Roth der »Versuchung, sie [...] auf das Liederbuch zu beziehen « und dabei Gefahr zu laufen, »Eisler aus der Perspektive Adornos zu deuten « (166, 168). Anstelle der Material-VerfahrensweiseThese wählt Roth eine von Eisler zweifelsfrei hochgehaltene Kategorie als Ausgangspunkt seiner Betrachtungen: Stimmigkeit, hervorgehend »aus der Konstruktion des Ganzen«. Inwieweit freilich die Forderung nach Stimmigkeit, insofern sie eine starke Betonung des Verfahrens (der 'Konstruktions) gegenüber dem Material impliziert, in der Praxis auf dasselbe oder zumindest sehr Ähnliches hinausläuft wie die Material-VerfahrensweiseThese, wird vom Autor nicht unmittelbar thematisiert; seine Analysen scheinen es indes zu bestätigen.

In zumindest einer Hinsicht lässt sich der Autor doch darauf ein, Eisler aus der Perspektive Adornos zu deuten. Sah letzterer die beobachtete »Vergleichgültigung des Materials» im Zusammenhang mit den Erfahrungen der Zwölftontechnik, so verschreibt sich Roth der "Idee, Eislers besonderen Umgang mit Tonalität im Hollywood-Liederbuch von der Dodekaphonie her zu erschließen« (114). Letztlich folgt er diesem Vorsatz aber nicht besonders stringent, und man hat am Ende das Gefühl, mehr über die Tonalitätsbehandlung in bestimmten exemplarisch untersuchten dodekaphonen Kompositionen Eislers erfahren zu haben - darüber allerdings sehr Anregendes - als umgekehrt über das Wirken dodekaphon geprägter Denkweisen in dessen Umgang mit Tonalität, das Phänomen der `Vertikalisierung، von Tonfolgen einmal ausgenommen. Nichtsdestoweniger gelingt Roth in einzelnen Analysen die Beschreibung, wie tonale Momente 
zum Material des Konstruierens eines tonalitätsunabhängig stimmigen Ganzen werden, mustergültig. Insofern solches Konstruieren musikgeschichtlich gesehen aber durchaus keine originäre Errungenschaft zwölftönigen Komponierens darstellt, sondern dort bestenfalls zu sich selbst kommt, hätte es des nominellen Rückbezugs auf die Dodekaphonie eigentlich gar nicht bedurft.

Hinsichtlich der Problematisierung des eigenen analytischen Instrumentariums macht Roth auch beim Begriff der Stimmigkeit keine Ausnahme. Dieser sei eine zwiespältige analytische Kategorie; denn "zu groß ist die Gefahr, auf einem einmal eingeschlagenen Weg bestimmte Aspekte auszublenden, die in andere Richtungen weisen oder nicht saufgehen « (168). Dass diese Gefahr besonders bei einem auf immanenten Beziehungsreichtum abhebenden Untersuchungsansatz wie der Grundgestalt-Analyse droht, dafür liefert wiederum der Autor selbst ein Anschauungsbeispiel, nämlich bei der Betrachtung des Liedes Die Weiden, wo, wie er ausführt, ein fünftöniger Akkord als Vertikalisierung aus der als Grundgestalt identifizierten Tonfolge abgeleitet sei (137). Allerdings muss er einräumen, dass ein Akkordton (fis) in der Grundgestalt nicht enthalten ist. Kann er diesen Ton noch als Rückgriff auf den ersten nach Ablauf der Grundgestalt erklingenden Melodieton rettenı, so schweigt er sich andererseits darüber aus, nach welchen Kriterien die akkordische Auswahl von vier der sechs Tonqualitäten der Grundgestalt erfolgt sein mag - zwei ihrer Töne, dis und $c$, fehlen im Akkord. Da sich der Nachweis einer Vertikalisierung somit als anfechtbar erweist, leidet auch die Überzeugungskraft von Roths Leitidee, Eislers Umgang mit Tonalität von der Dodekaphonie her zu erschließen.

Die wohl schwierigste Aufgabe, die Roth sich stellt, besteht in der Einbeziehung der semantischen Ebene in die Analyse; allerdings formuliert er diesbezüglich auch einen derart hohen Anspruch, dass er fast zwangsläufig dahinter zurückbleiben muss: Seinem Ansatz, so der Autor, liege die »Prämisse zugrunde, dass inhaltliche Aspekte nur von den inneren
Gesetzmäßigkeiten des musikalischen Formprozesses her erschlossen werden können« (153). In der Umsetzung läuft dieses ErschlieBen wiederholt auf die formelhafte Lösung hinaus, dass Gehalte auf einen Grundwiderspruch oder einen grundlegenden gestischen Kontrast oder Umschlag zurückgeführt werden, für den sich dann eine Schärfung durch die Musik dingfest machen lässt (siehe die im Übrigen hervorragenden Analysen von Frühling und An die Hoffnung, 149-164). Freilich nähert sich Roth den Fragen der Musik-InhaltVermittlung noch auf anderen Wegen. Zu nennen ist hier zum einen die Auseinandersetzung mit intertextuellen Bezügen im Liederbuch (sowohl auf eigene Werke als auch z. B. auf Lieder von Schubert und Schumann), zum anderen das Eingehen auf Eislers Mobilisierung satztechnischer Topoi mit den an ihnen haftenden Bedeutungen (darunter fällt im Prinzip auch die bereits erwähnte 'Kontextualisierung ` von Ausdrucksgehalten).

Was die auf die musikalische Tradition bezogene Intertextualität betrifft, spürt Roth insbesondere einigen subtilen kompositorischen Referenzen auf Schumanns Liederkreis op. $39 \mathrm{im}$ Lied Erinnerung an Eichendorff und Schumann nach, doch steht am Ende die Erkenntnis, dass derartige Anspielungen von Eisler eher als Privatangelegenheit behandelt wurden; der Autor spricht in diesem Zusammenhang von einer »Ästhetik der Spurenverwischung" sowie einer »Poetik des Enigmatischen « $(243,262)$. Auch die Untersuchung von Eislers Querverweisen aufs eigene OEuvre erweist sich als nicht allzu ergiebig. Zwar kann Roth mit der nicht uninteressanten Entdeckung aufwarten, dass in zwei Stücken des Liederbuchs auf eine mottohafte Akkordfortschreitung aus den Zeitungsausschnitten op. 11 von 1926 zurückgegriffen wird, muss aber zugleich eingestehen, dass zwischen diesen drei Werken inhaltlich »keinerlei verbindende Bezüge auszumachen« sind (262). Konkretere Aussagen ermöglicht demgegenüber die Konzentration auf die Verwendung satztechnischer Topoi, namentlich Fauxbourdonsatz, Lamento-Bass und Neapolitanischer Sextakkord bzw. sneapolitanische Färbung;; 
im Ganzen jedoch bleibt die semantische Ebene im Verhältnis zu den im engeren Sinn kompositionstechnischen Fragen eher unterbelichtet. Hier scheint Roth ein grundsätzlich vorhandenes Potenzial verschenkt zu haben. Seinem an sich sehr klugen Ansatz einer Liedanalyse, die von der Erkenntnis ausgeht, dass bei Eisler nicht »jedes Schlüsselwort an Ort und Stelle ein Klangsymbol hervorruft" (55), hätte eine bewusstere und gezieltere systematische Anwendung des Begriffs des 'Gestischen vermutlich gute Dienste leisten können. Dieser taucht auf Seite 156 wie aus dem Nichts und danach nur sporadisch auf als Allerweltsvokabel, ohne dass seine Rolle in Brechts Theatertheorie thematisiert wird.

Als seine Leitidee bezeichnet Roth in der Einleitung, „das für Eislers CEuvre in verschiedenster Hinsicht grundlegende Prinzip der Collage sowohl auf die Gliederung als auch auf die Darstellung des Gegenstands zu übertragen« (16). Man hätte dem Autor den Mut gewünscht, das Konzept einer lockeren sanalytischen Collager konsequenter und offensiver umzusetzen; im vorliegenden Ergebnis jedoch reibt es sich merklich mit dem Versuch, nach außen der Erwartung eines >roten Fadens« Genüge zu tun. Jede einzelne der mit scharfem Verstand, historischer Kompetenz, philologischer Genauigkeit und musikalischer Sensibilität verfassten Analysen lohnt unbedingt die Lektüre; die Konzeption ihrer Zusammenstellung dagegen wirkt zuweilen aufgepfropft. So rücken in einem Kapitel, das "Grenzbereiche der schwebenden Harmonie« überschrieben ist, überraschenderweise fundamentale Überlegungen zu Fragen der Semantik in den Vordergrund, und eine als Exkurs eingeschobene, fraglos studierenswerte Analyse von Eislers dritter Klaviersonate wird in solcher Detailliertheit durchgeführt, dass ihre Relevanz für den erklärten Gegenstand der Studie aus dem Blick zu geraten droht. Leider wirken sich die Irritationen des Aufbaus negativ auf die Zugänglichkeit der Arbeit aus. Der Leser, der sich durch ihre Gliederung herausgefordert fühlt, Zusammenhänge auch dort zu erfassen, wo möglicherweise keine sind, vermeint, wenn er sie nicht findet, etwas überlesen oder nicht verstanden zu haben, was die Lektüre mühseliger macht als sie sein müsste. (Beim Schreiben musikalischer Analysen stets die Gewichtung der Einzelbefunde im Hinblick auf eine immer im Auge zu behaltende Generallinie transparent zu machen, ist vielleicht das Kernproblem dieser Textsorte.)

All dies sind freilich nebensächliche Einwände in Anbetracht der Tatsache, dass Roth beim Abbau eines in der Eisler-Forschung grundsätzlich zu konstatierenden Musiktheorie-Defizits Pionierarbeit geleistet und die Einsicht in Eislers kompositorisches Denken entscheidend vertieft hat. Mit ihrem Eingehen auf vokale Musik bildet seine Studie in dieser Hinsicht ein wertvolles Komplement zu Thomas Ahrends 2006 erschienener Arbeit Aspekte der Instrumentalmusik Hanns Eislers. Durch die Anstrengungen beider ist eine fundierte Bestimmung der Position Eislers innerhalb der Neuen Wiener Schule bedeutend leichter geworden. Aber auch über diesen Kontext hinaus verdient Der Gesang als Asyl Beachtung - mit der Identifizierung und Beschreibung von Techniken, die eines der zentralen Probleme in der Musik des 20. Jahrhunderts angehen: die Idee des Musizierens über Musik.

Tobias Fasshauer 